\title{
CALISTEGINAS, ALCALÓIDES POLIHIDROXILADOS PRESENTES EM HORTALIÇAS: FRUTOS E TUBEROSAS
}

\author{
Luís Fernando L. Souto ${ }^{\mathrm{a}, *, \text { (i) e Antonio Jorge Ribeiro da Silva }}{ }^{\mathrm{b}}$ \\ a'Departamento de Apoio ao Ensino, Instituto Federal de Educação, Ciência e Tecnologia de Rondônia Câmpus Porto Velho Calama \\ 76820-441 Porto Velho - RO, Brasil \\ ${ }^{\mathrm{b}}$ Instituto de Pesquisas de Produtos Naturais, Universidade Federal do Rio de Janeiro 21941-902 Rio de Janeiro - RJ, Brasil
}

Recebido em 15/06/2019; aceito em 28/08/2019; publicado em 04/12/2019

\begin{abstract}
CALYSTEGINES, POLYHYDROXYLATED ALCALOIDS PRESENT IN VEGETABLES: FRUITS AND TUBERS. Calystegines are nortropane alkaloids with three to five hydroxyl groups. These alkaloids are recognized as glycosidase inhibitors and thus may have therapeutic potential to be exploited. There are also reports of its possible toxic effects when they are present in foods. Commercial cultivars of human consumption in Brazil have been analyzed for calystegines content. Samples of Solanum tuberosum L. (potato), Ipomoea batatas L. (sweet potato), Solanum lycopersicum (tomato), Solanum melongena (eggplant) and Solanum aethiopicum L. (gilo) were obtained from local markets in Rio de Janeiro, RJ, Brazil. Qualitative analyzes were performed by gas chromatography coupled to mass spectrometry (GC/MS) and high performance liquid chromatography coupled to mass spectrometry (LC/MS). Gas chromatography with flame ionization detector (GC/FID) was used in quantitative analysis. Five calystegines $\left(\mathrm{A}_{3}, \mathrm{~B}_{1}\right.$, $\mathrm{B}_{2}, \mathrm{~B}_{4}$ and $\mathrm{C}_{1}$ ) were detected in the whole sample set and the amounts of three of them were determined: $\mathrm{A}_{3}\left(1.94-15.88 \mathrm{mg} \mathrm{kg}^{-1}\right) ; \mathrm{B}_{2}$ (7.59-23.7 $\left.\mathrm{mg} \mathrm{kg}^{-1}\right)$ and $\mathrm{B}_{4}\left(3.61-9.38 \mathrm{mg} \mathrm{kg}^{-1}\right)$. This is the first report on the occurrence of these alkaloids in Solanum aethiopicum $\mathrm{L}$.
\end{abstract}

Keywords: commercial cultivars; calystegine alkaloids; glycosidase inhibitors.

\section{INTRODUÇÃo}

As calisteginas constituem um grupo de alcaloides nortropânicos que contém de três a cinco grupos hidroxila. Devido a essa característica estrutural elas receberam a seguinte classificação: as calisteginas do tipo A contêm três grupos - $\mathrm{OH}$; as do tipo B quatro e as do tipo $\mathrm{C}$ cinco. Além destas, foram identificadas calisteginas $\mathrm{N}$-metiladas, glicosiladas e a calistegina $\mathrm{N}$, que tem um grupo amino ligado à cabeça de ponte. ${ }^{1}$ Estes alcaloides tem estrutura similar à de açúcares, e esta similaridade estrutural pode ser o que faz com que interajam com glicosidases, levando à inibição do metabolismo de carboidratos. ${ }^{2}$

A ocorrência desses metabólitos tem sido relatada nas famílias Convolvulaceae, Solanaceae, Moraceae, Erythroxylaceae, Brassicaceae e Ericaceae. ${ }^{3-8}$ Assim, calisteginas já foram detectadas em batatas doces (Ipomoea batatas), batatas (Solanum tuberosum L.), tomates (Solanum lycopersicum), pimentões (Capsicum Anuum), berinjelas (Solanum melongena) dentre outras espécies alimentícias. ${ }^{9}$ Essas plantas são consumidas em muitas partes do mundo e, no caso das batatas, elas são uma importante fonte de carboidratos. Se as calisteginas agem nas glicosidases intestinais, é concebível que estes vegetais, quando presentes na dieta humana inibam ou retardem a digestão de carboidratos. ${ }^{2}$

Embora mais de 200 alcaloides tropânicos e nortropânicos diferentes tenham sido identificados em várias plantas, os respectivos dados sobre sua toxicidade e ocorrência em alimentos são limitados. ${ }^{10}$ Na Europa os níveis de alcaloides tropânicos e nortropânicos em alimentos já são uma preocupação. A Autoridade Europeia para a Segurança dos Alimentos (European Food Safety Authority - EFSA) publicou em 2016 resultados de um estudo sobre a ocorrência de alcaloides tropânicos em alimentos em nove países europeus. ${ }^{11}$ Recentemente a EFSA também estimou a exposição alimentar à essas substâncias em todas as classes etárias, empregando um banco

*e-mail: luis.lira@ifro.edu.br de dados de ocorrência desses metabólitos em um conjunto amplo de categorias de alimentos. ${ }^{10}$

No Brasil ainda não há registro de trabalhos voltados para a determinação de calisteginas em espécies alimentícias. Portanto, o presente estudo busca determinar a ocorrência desses metabólitos em algumas cultivares de vegetais que fazem parte da dieta do brasileiro, tendo em vista que, o interesse prático nessa classe de substâncias concentra-se principalmente em duas áreas: são inibidores de glicosidases com potencial terapêutico e são constituintes de alimentos e rações com possíveis efeitos tóxicos. ${ }^{12}$

\section{PARTE EXPERIMENTAL}

\section{Padrões e reagentes}

Os solventes utilizados na extração e nas análises por CG/EM e CG/FID foram todos de elevado grau de pureza. A resina de troca iônica (Dowex AG 50W X8), o meio filtrante (Celite 545), o reagente para derivatização $N$-metil- $N$-trimetilsililtrifluoroacetamida (MSTFA) e a piridina anidra foram adquiridos da Sigma Aldrich. O perseitol (D-glycero-D-galacto-heptitol) da Santa Cruz Biotechnology e os padrões de calisteginas $\mathrm{A}_{3}, \mathrm{~B}_{2}$ e $\mathrm{B}_{4}$ da Carbosynth. Os demais reagentes utilizados são de alta pureza.

\section{Coleta das amostras}

A partir de uma revisão na literatura sobre a ocorrência de calisteginas em espécies alimentícias foram selecionadas para análise as seguintes espécies (cultivar - cv): batatas, Solanum tuberosum L. (cv asterix); batatas doces, Ipomoea batatas L. (cv BRS rubissol); tomates, Solanum lycopersicum (cv BRS imigrante); berinjelas, Solanum melongena (cv picola); e jilós, Solanum aethiopicum L. (cv comprido verde claro e cv morro redondo).

As amostras foram adiquiridas em estabelecimentos comercias escolhidos aleatoriamente na cidade do Rio de Janeiro (Brasil) e 
a amostragem foi feita nas condições em que o produto chega ao consumidor final. As cultivares foram coletadas duas vezes, entre março e novembro de 2018, sendo recolhido $1 \mathrm{~kg}$ de cada material por amostragem.

\section{Extração das calisteginas}

As etapas de extração e derivatização seguiram a metodologia empregada por Friedman et al. (2003) com algumas alterações. ${ }^{13}$

O material fresco foi triturado, homogeneizado e em seguida pesado $1 \mathrm{~g}$ em triplicata. A extração foi realizada com metanol/água $(1: 1, \mathrm{v} / \mathrm{v}, 20 \mathrm{~mL})$ em banho ultrassônico por $30 \mathrm{~min}$. Cada amostra foi filtrada sob pressão reduzida através de meio filtrante (Celite 545), e o filtrado foi reduzido para $3 \mathrm{~mL}$ utilizando evaporador rotatório a $60{ }^{\circ} \mathrm{C}$ sendo o pH ajustado para 4,0 com $\mathrm{HCl} 1 \mathrm{M}$. A solução foi introduzida em uma coluna (50 mm x $12 \mathrm{~mm}$ ) empacotada com $15 \mathrm{~mL}$ de resina de troca iônica de caráter ácido (Dowex AG 50W X8) e lavada três vezes com água ultrapura. Em seguida o material foi eluído com solução aquosa de $\mathrm{NH}_{4} \mathrm{OH} 2 \mathrm{M}$, utilizando vazão de aproximadamente $1 \mathrm{~mL} \mathrm{~min}{ }^{-1}$. O eluente alcalino foi recolhido e o volume reduzido para 2-6 mL, utilizando evaporador rotatório a $60^{\circ} \mathrm{C}$. A solução resultante foi transferida para um balão volumétrico de $10 \mathrm{~mL}$, diluída e avolumada com água ultrapura. Alíquotas de 1,0 mL foram transferidas para frascos (vials) de $1,5 \mathrm{~mL}$ e liofilizadas.

\section{Derivatização}

Calisteginas não são voláteis, nem lipofílicas, assim requerem transformação para uma forma volátil com agentes de sililação suaves antes da análise por CG. ${ }^{1}$ Deste modo, a cada vial contendo o extrato alcaloídico foram adicionados $50 \mu \mathrm{L}$ de piridina anidra, $45 \mu \mathrm{L}$ $\mathrm{N}$-metil-N-trimetilsililtrifluoroacetamida (MSTFA), a reação segue ilustrada na Figura 1. Como padrão interno $5 \mu \mathrm{L}$ de uma solução de $2 \mathrm{mg} \mathrm{mL}^{-1}$ de perseitol (D-glicerol-D-galacto-heptitol) derivatizado com $50 \mu \mathrm{L}$ de piridina anidra e $50 \mu \mathrm{L}$ MSTFA previamente aquecidas durante $1 \mathrm{~h}$ a $100{ }^{\circ} \mathrm{C} .{ }^{13}$ Os frascos foram então aquecidos por $30 \mathrm{~min}$ a $60^{\circ} \mathrm{C}$ em um banho seco para frascos (vials) de $1,5 \mathrm{~mL}$.

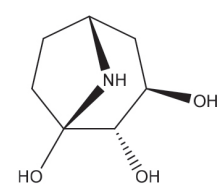

Calistegina $\mathrm{A}_{3}$

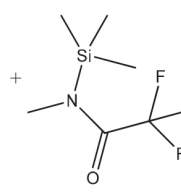

MSTFA

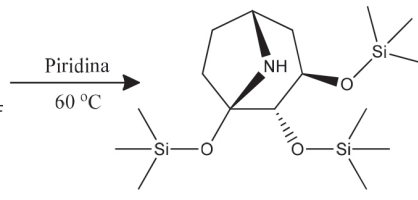

Calistegina $\mathrm{A}_{3}$-TMS
Figura 1. Equação para a reação de derivatização da calistegina $A_{3}$ com MSTFA

\section{Análise cromatográfica}

Cromatografia em fase gasosa acoplada à espectrometria de massas (CG/EM)

Nas análises cromatográficas foi utilizada a metodologia empregada por Bourebaba et al. (2016) modificada. ${ }^{14}$ As amostras derivatizadas foram analisadas em um cromatógrafo CG/EM (GCMS-QP2010 Plus Shimadzu), equipado com uma coluna DB-5MS (30 m x 0,25 mm x 0,25 $\mu \mathrm{m}$ ), empregando He como gás de arraste e velocidade linear de $37 \mathrm{~cm} \mathrm{seg}^{-1}$. O volume injetado foi $1 \mu \mathrm{L}$ no modo divisão de fluxo (razão 1:20) com temperatura do injetor de $250^{\circ} \mathrm{C}$ e programação de temperatura iniciando com $100^{\circ} \mathrm{C}$ durante 5 min, seguida por uma taxa de aquecimento de $10^{\circ} \mathrm{C} \mathrm{min}{ }^{-1}$ até $270{ }^{\circ} \mathrm{C}$, temperatura que foi mantida por $5 \mathrm{~min}$. O tempo total de análise foi de 27 min. Os parâmetros de detecção no espectrômetro de massas foram: temperatura da interface $280{ }^{\circ} \mathrm{C}$ e da fonte de íons, $200{ }^{\circ} \mathrm{C}$; faixa de massas, $m / z$ 50-600; eletroionização a $70 \mathrm{eV}$, velocidade de varredura 1428 amu.seg ${ }^{-1}$; tempo de evento 0,50 seg. As amostras foram analisadas simultaneamente nos modos de aquisição $S C A N$ (full scan mode) e SIM (selective ion monitoring mode).

Para a identificação das calisteginas a partir dos cromatogramas obtidos no modo SIM foram monitorados os seguintes pares de íons: $129 \mathrm{~m} / \mathrm{z}$ e $244 \mathrm{~m} / \mathrm{z}$ para $\mathrm{B}_{1} ; 217 \mathrm{~m} / \mathrm{z}$ e 229 para $\mathrm{B}_{2} ; \mathrm{m} / \mathrm{z}, 217$ e 259 para $\mathrm{B}_{3} ; \mathrm{m} / \mathrm{z} 217$ e 244 para $\mathrm{B}_{4} ; \mathrm{m} / \mathrm{z} 217$ e 375 para $\mathrm{C}_{1} ; \mathrm{m} / z 244$ e $156 \mathrm{~m} / \mathrm{z}$ para $\mathrm{A}_{3}$ e $\mathrm{A}_{5 ;}$ e $\mathrm{m} / \mathrm{z} 390$ para $\mathrm{N}_{1} \cdot{ }^{14}$ A coleta e processamento dos dados foram realizadas utilizando o software GCMS Solution versão 2.5 (Shimadzu).

Os tempos de retenção e espectros de massas de padrões autênticos foram utilizados para confirmar a presença das calisteginas $\mathrm{A}_{3}$, $\mathrm{B}_{2}$ e $\mathrm{B}_{4}$, como éteres de TMS nas amostras. Para a mesma finalidade, também foram utilizados espectros de massas destas substâncias publicados na literatura para estas e para as demais calisteginas. ${ }^{15-17}$

\section{Cromatografia em fase gasosa com detecção por ionização em chama (CG/FID)}

As amostras foram analisadas por cromatografia em fase gasosa com detecção por ionização em chama em um cromatógrafo com detector FID (CG-2010 Plus Shimadzu), equipado com um injetor automático (AOC-20) e uma coluna DB-5MS (30 m x 0,25 mm x 0,25 $\mu \mathrm{m}$ ). As condições cromatográficas foram as mesmas empregadas nas análises por CG/EM, com a temperatura do detector em $300{ }^{\circ} \mathrm{C}$, velocidade linear do gás de arraste $30 \mathrm{~cm} \mathrm{seg}^{-1}$. Curvas de calibração foram construídas a partir de padrões analíticos de calisteginas $\mathrm{A}_{3}, \mathrm{~B}_{2}$ e $\mathrm{B}_{4}$ (Carbosynth) com uma série de seis (06) soluções de calisteginas trimetilsililadas em concentrações variando de 0,01 a $0,2 \mathrm{mg} \mathrm{L}^{-1}$. As concentrações desses três alcaloides foram calculadas a partir das áreas de pico dos analitos pelo método do padrão interno (perseitol-TMS).

\section{Cromatografia em fase líquida de alta eficiência acoplada à espectrometria de massas (CLAE/EM)}

A análise por cromatografia em fase líquida de alta eficiência (CLAE) foi realizada em um sistema Dionex UltiMate 3000 (Thermo Scientific). A análise cromatográfica foi realizada em modo HILIC (Cromatografia Líquida com Interação Hidrofílica), nas separações empregou-se uma coluna ACE HILIC-A 100 x $2.1 \mathrm{~mm}$ de diâmetro e partículas de $1.7 \mu \mathrm{m}$. A fase móvel foi composta por água (fase A) e acetonitrila (fase B), ambos com $5 \mathrm{mM}$ de formiato de amônio. Nas análises foi empregado o modo de eluição isocrático com $5 \%$ de A e $95 \%$ de B, vazão $0,3 \mathrm{~mL} / \mathrm{min}$ e temperatura do forno $25^{\circ} \mathrm{C}$. Amostras foram injetadas usando um amostrador automático TriPlus RSH (Thermo Scientific) com volume de injeção de $5 \mu \mathrm{L}$.

O sistema CLAE foi acoplado a um espectrômetro de massas QExactive Plus (Thermo Scientific) de alta resolução, equipado com uma fonte de íons electrospray operando no modo positivo. Os parâmetros de ionização da fonte foram: tensão de eletrovaporização de 3,8 kV; temperatura capilar $300{ }^{\circ} \mathrm{C}$; S-Lens nível 70, GS1 40 e GS2 25. As amostras foram analisadas na faixa de varredura de $\mathrm{m} / z, 120$ a 600 na resolução de 70000 (varredura completa positiva) seguida por MS $^{2}$ usando resolução de 17500 e a energia de colisão normalizada (NCE) aumentando de 35-50\%. Os cromatogramas foram processados usando o software X calibur $^{\mathrm{TM}}$ versão 3.0.

\section{RESULTADOS E DISCUSSÃO}

\section{Ocorrência de calisteginas nas amostras}

As calisteginas detectadas em todo o conjunto amostral foram: $\mathrm{A}_{3}, \mathrm{~B}_{1}, \mathrm{~B}_{2}, \mathrm{~B}_{4}$ e $\mathrm{C}_{1}$ (Figura 2). 


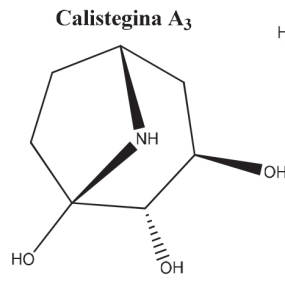

Calistegina $\mathbf{B}_{4}$
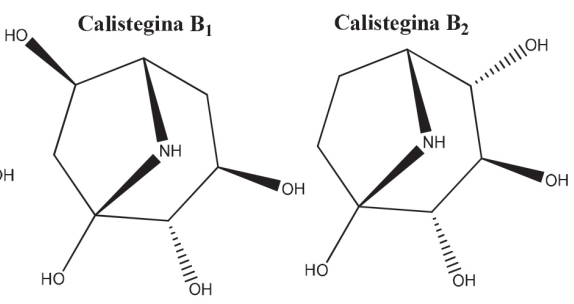

Calistegina $\mathbf{C}_{1}$

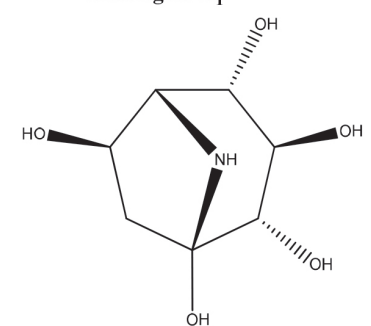

Figura 2. Estrutura das calisteginas detectadas nas amostras analisadas

Cromatogramas reconstruídos típicos das amostras obtidos por CG/EM no modo de aquisição SIM, para os padrões $\left(0,05 \mathrm{mg} \mathrm{mL}^{-1}\right)$, batatas asterix e batatas doces estão ilustrados na Figura 2. As calisteginas $\mathrm{A}_{3}, \mathrm{~B}_{2}$ e $\mathrm{B}_{4}$ foram identificadas por comparação dos seus tempos de retenção relativos ao perseitol e seus espectros de massas com os obtidos de padrões autênticos. Além do auxílio dos dados adquiridos em modo de aquisição $S I M$ via CG/EM, as demais calisteginas também foram identificadas por comparação com padrões de fragmentação (Figura 3) publicados na literatura. ${ }^{16-18}$

Cabe destacar que o uso do modo SIM aumenta a sensibilidade e seletividade do método, ${ }^{19}$ possibilitando a realização de análises qualitativas em conjunto com os espectros de massas obtidos pelo modo SCAN (Figura 4). Com esses dados em mãos foi possível identificar a presença das calisteginas $\mathrm{B}_{1}$ e $\mathrm{C}_{1}$ sem a necessidade do uso de padrões analíticos, tendo em vista que são substâncias conhecidas com perfil de fragmentação divulgado na literatura. ${ }^{16-18}$

O sinal correspondente a $\mathrm{m} / \mathrm{z} 73$ está presente em todos os espectros de massas (Figura 4), com elevada intensidade (50-100\%) e corresponde ao fragmento trimetilsilil (TMS). À semelhança do que ocorre com os espectros de massas de carboidratos, obtidos por meio de eletroionização, no caso das calisteginas extensa fragmentação acontece após a ionização desfavorecendo o aparecimento dos picos moleculares em virtude da sua baixa abundância relativa na corrente iônica. No caso dos carboidratos, é prática comum a utilização de pares de fragmentos iônicos característicos como auxiliares para a identificação das substâncias nos seus espectros $S I M .{ }^{20}$ No presente trabalho, os íons moleculares não são observados no espectro devido suas baixas abundâncias relativas. São raros os estudos sistemáticos da fragmentação de calisteginas. ${ }^{18}$ Nos espectros obtidos, apesar de sua pouca intensidade $(<1 \%)$ é possível notar as perdas referentes a um fragmento neutro de 15 unidades de massa $\left(\mathrm{CH}_{3}\right)$ para $\mathrm{B}_{1}, \mathrm{~B}_{2}$ e $\mathrm{B}_{4}(\mathrm{~m} / \mathrm{z} 448)$ e de fragmento de 90 unidades de massa (HOTMS) para $\mathrm{A}_{3}(\mathrm{~m} / \mathrm{z} 285)$ e $\mathrm{C}_{1}(\mathrm{~m} / \mathrm{z} 461)$. Em relação às calisteginas do grupo A, $m / z, 156$ é o fragmento de maior intensidade, o qual resulta da cisão do biciclo (Figura 5). O pico base $m / z, 217$ indica a presença de três grupos O-TMS adjacentes de calisteginas derivatizadas, uma fragmentação característica de calisteginas dos grupos B e C, com exceção da calistegina $B_{1}$ cujos fragmentos $m / z, 129$ e $m / z, 244$ são os mais intensos (Figura 5). ${ }^{18}$

Visando consolidar os dados obtidos via GC/EM para as calisteginas $\mathrm{B}_{1}$ e $\mathrm{C}_{1}$, devido a ausência de padrões analíticos, a detecção desses analitos também foi realizada por CLAE/EM. Os padrões de fragmentação (Figura 6) foram comparados com a literatura, ${ }^{21}$ reforçando as evidências da presença dessas duas calisteginas nos extratos.

Deste modo, calisteginas foram detectadas em todas as cultivares analisadas. Na Tabela 1 constam as ocorrências detectadas por cultivar analisado.

Como esperado, $\mathrm{B}_{2}$ e $\mathrm{A}_{3}$ estavam presentes na maioria das cultivares, são comuns em batatas e tomates, também já haviam sido encontradas em berinjelas..$^{9,22}$ Os resultados obtidos para batatas asterix estão em concordância com dados publicados por Petersson $e t$ al. (2013) que detectaram $\mathrm{A}_{3}, \mathrm{~B}_{2}$ e $\mathrm{B}_{4}$ nessa cultivar. ${ }^{17}$ As calisteginas encontradas em berinjelas $\left(\mathrm{A}_{3}, \mathrm{~B}_{1}\right.$ e $\left.\mathrm{B}_{2}\right)$ foram também reportadas por Nash et al. (1993). ${ }^{22}$ Asano et al. (1997) encontraram apenas calisteginas $\mathrm{B}_{1}$ e $\mathrm{B}_{2}$ nesta espécie, ${ }^{9}$ contudo, seus resultados para

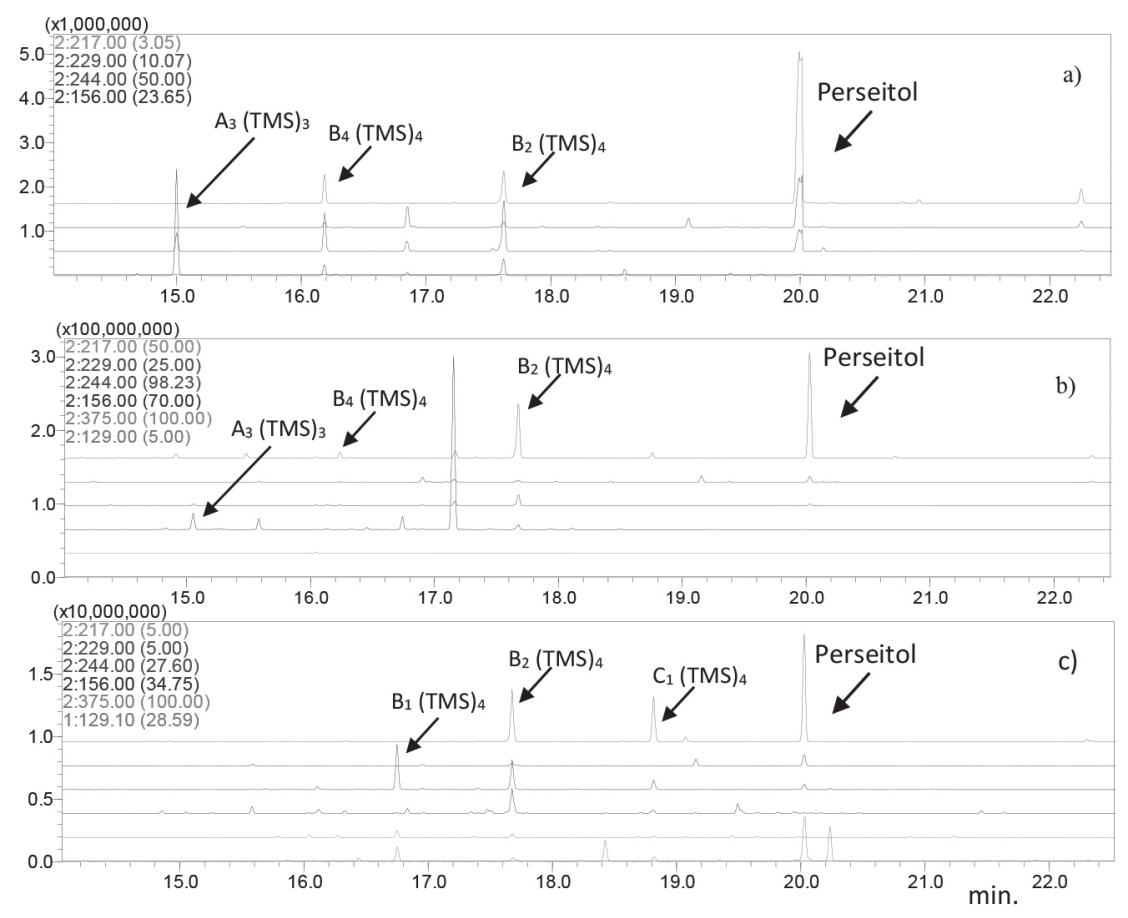

Figura 3. Cromatogramas CG/EM (SIM) de trimetilsilil (TMS) derivados de calisteginas obtidos para: a) Padrões $A_{3}, B_{2}$ e $B_{4}$; b) batatas asterix e c) batatas doces 

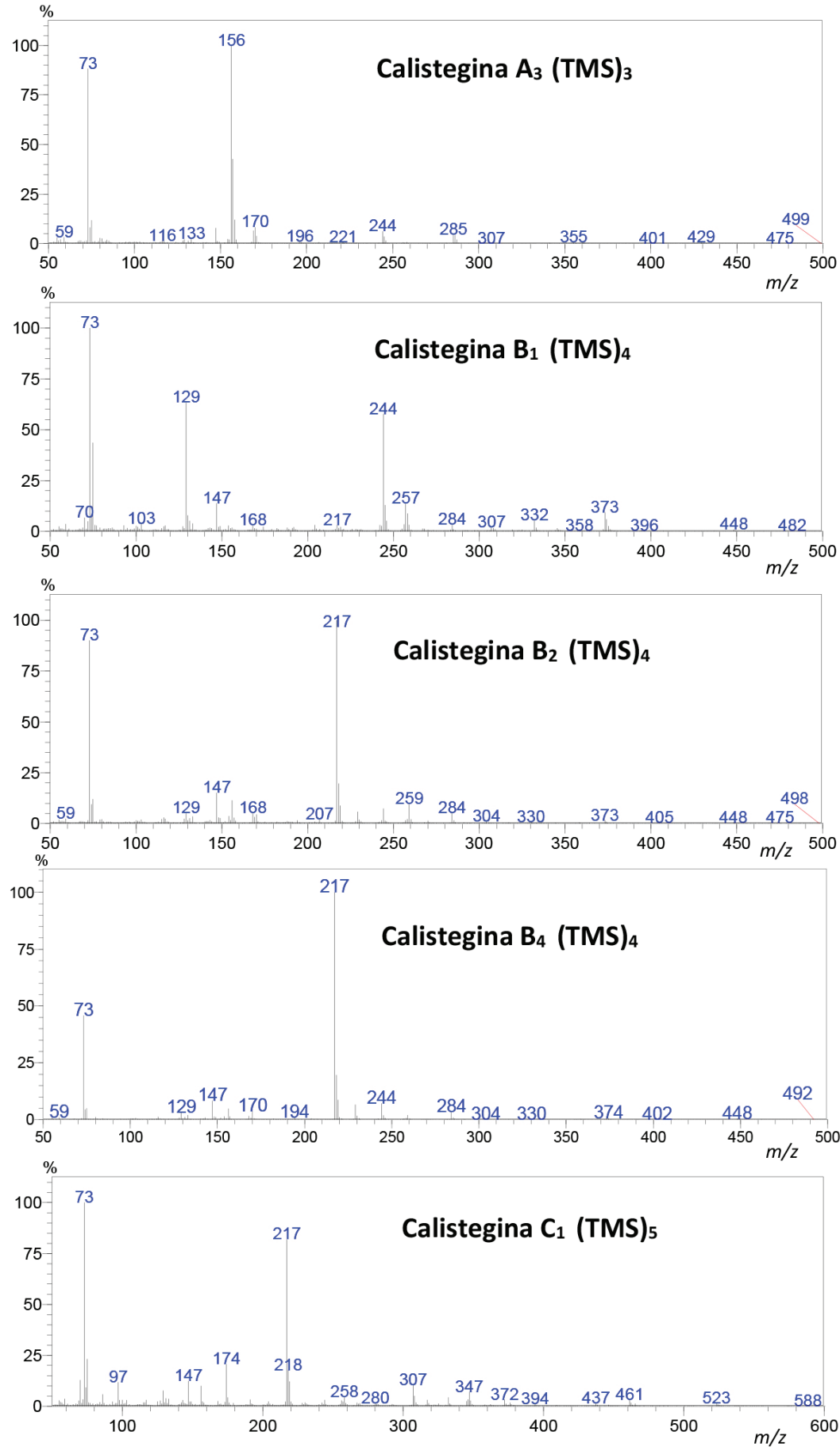

Figura 4. Espectros de massas obtidos para trimetilsilil derivados das calisteginas $A_{3}, B_{1}, B_{2}, B_{4}$ e $C_{1}$ em amostras

tomates e batatas doces coincidem com os obtidos no presente estudo. Cabe ainda ressaltar que, essa é a primeira vez em que é relatada a ocorrência desses metabólitos em jilós (Solanum aethiopicum L.).

\section{Análise quantitativa}

As calisteginas $A_{3}, B_{2}$ e $B_{4}$ foram quantificadas com uso de padrões analíticos por CG/FID, as curvas foram construídas com uma série de seis (06) soluções de calisteginas trimetilsililadas com concentrações variando de 0,01 a $0,2 \mathrm{mg} \mathrm{L}^{-1}$, a linearidade foi demonstrada por valores de coeficientes de correlação superiores a 0,98 nas curvas para os três analitos (Tabela 2). O limite de detecção (LD) e o limite de quantificação (LQ) foram determinados pelo método baseado em parâmetros da curva analítica, o qual é apontado como o melhor caminho para o cálculo do LD e LQ em cromatografia, por ser estatisticamente mais confiável. ${ }^{23}$

Após a confecção da curva as triplicatas das amostras foram analisadas, os resultados obtidos estão descritos Tabelas 3 e 4 em termos de média e desvio padrão de concentração em massa fresca do material.

Além de estar presente em todas as cultivares analisadas, a calistegina $\mathrm{B}_{2}$ também foi a majoritária, ocorrendo em concentrações médias entre 7,59-23,79 mg kg-1 apresentado a maior variação entre os dois lotes amostrados. As batatas asterix apresentaram os maiores teores desse alcalóide, equanto os tomates os menores. Essa predominânca frente às demais calisteginas está em concordância com a 

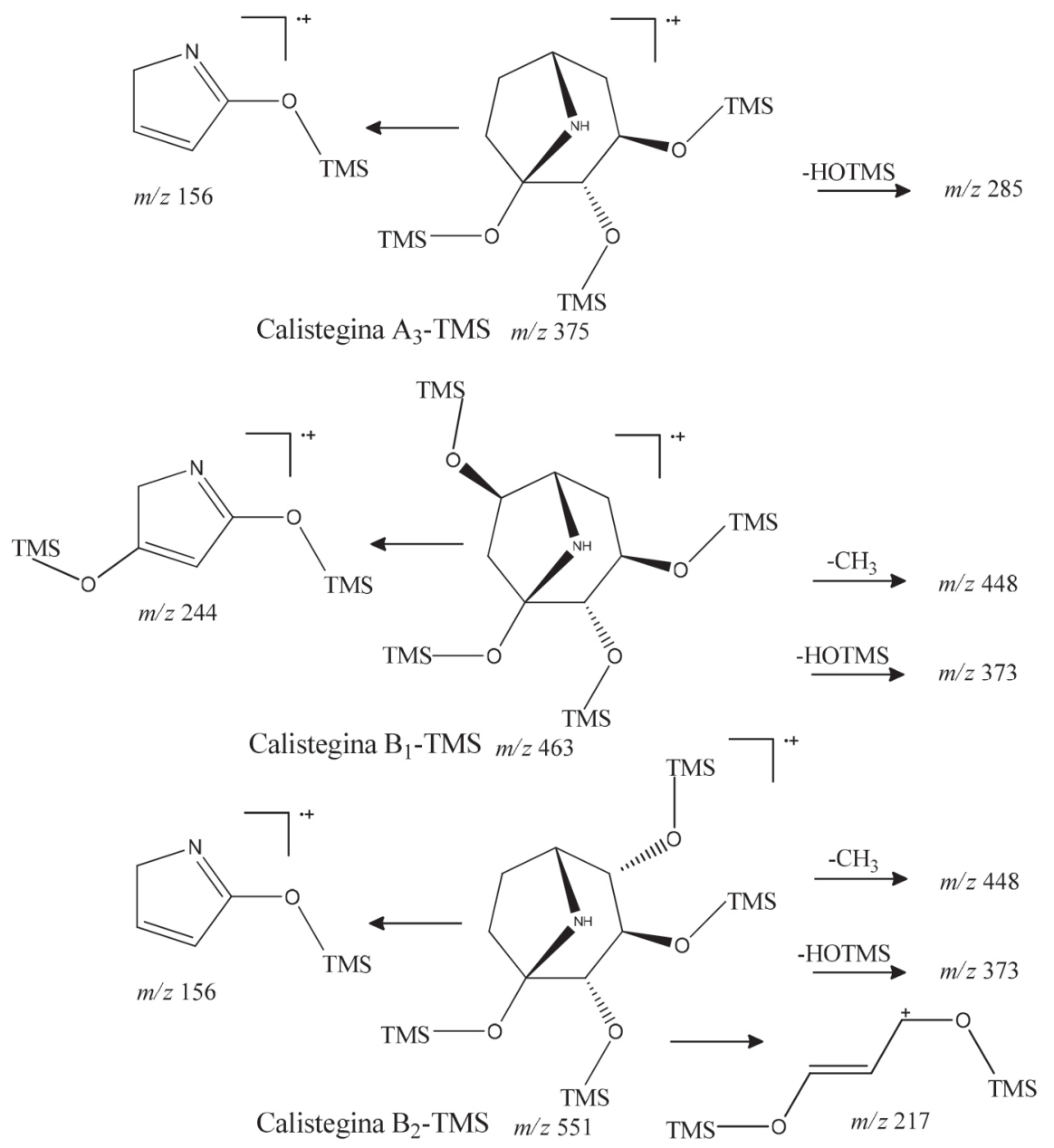

Figura 5. Fragmentação de calisteginas derivatizadas, eletroionização $(70 \mathrm{eV})^{18}$

literatura, tendo sido reportada em epécies das famílias Solanaceae, Convolvulaceae, Eritroxilaceae, Ericaceae, entre outras. ${ }^{6,8,24,25}$

Apesar de ter sido detectada na maioria das cultivares, as concentrações de calistegina $\mathrm{A}_{3}$ foram determinadas apenas em batatas axterix, jilós comprido verde claro e tomates imigrante, sua concentração variou entre $1,94-15,88 \mathrm{mg} \mathrm{kg}^{-1}$. Nas demais amostras onde sua presença havia sido detectada o sinal ficou abaixo do LQ. Assim como a $B_{2}$, o maior teor deste alcalóide foi encontrado em batatas asterix. A ocorrência de calistegina $B_{4}$ se deu apenas em batatas asterix, suas concentrações ficaram entre $3,61-9,38 \mathrm{mg} \mathrm{kg}^{-1}$, resultados corroborados pelos dados da literatura, onde se verifica que calisteginas $B_{2}, A_{3}$ e $\mathrm{B}_{4}$ são, nesta ordem, as mais comuns em batatas. ${ }^{13,17,26}$

As concentrações das calisteginas $\mathrm{A}_{3}, \mathrm{~B}_{2}$ e $\mathrm{B}_{4}$ em batatas asterix ficaram próximas dos valores encontrados por Petersson et al. (2013) em amostras desta mesma cultivar (30-69 mg kg-1 33-54 mg kg-1 $\mathrm{e}$ $5-4 \mathrm{mg} \mathrm{kg}^{-1}$ respectivamente) adquiridas de produtores e/ou no comércio local na Suécia. ${ }^{17}$ Os autores suecos submeteram suas amostras a condições extremas, com ferimentos mecânicos, exposição à luz ou temperatura elevada, simulando situações que estes produtos pudessem sofrer durante colheita e transporte, até sua chegada ao consumidor final. Contudo, as concentrações medidas ficaram dentro de uma ampla faixa que tem sido registrada em cultivares de batatas, que podem conter até $2300 \mathrm{mg} \mathrm{kg}^{-1}$ desses alcaloides por peso de massa fresca. ${ }^{26}$

Dentro do grupo de amostras em que foi possível determinar a concentração das calisteginas $\mathrm{A}_{3}$ e $\mathrm{B}_{2}$, os tomates, entre as hortaliças analisadas apresentaram o menor teor desses alcaloides. As concentrações ficaram próximas ao que foi encontrado em tomates japoneses:
$\mathrm{A}_{3}\left(1,1 \mathrm{mg} \mathrm{kg}^{-1}\right)$ e $\mathrm{B}_{2}\left(4,5 \mathrm{mg} \mathrm{kg}^{-1}\right) .{ }^{9}$ No entanto, distantes dos níveis medidos em derivados de tomates na Espanha: $\mathrm{B}_{2}\left(0,4-0,5 \mathrm{mg} \mathrm{kg}{ }^{-1}\right)$ e $\mathrm{A}_{3}\left(9,5-19 \mathrm{mg} \mathrm{kg}^{-1}\right){ }^{21}$

Nas berinjelas, que além de alimentícias são plantas com muitas propriedades medicinais, ${ }^{27}$ foi possível determinar a concentração apenas de calistegina $\mathrm{B}_{2}$, os valores encontrados estão dentro da faixa de concentração que tem sido observada na literatura para a espécie (0,5 - $\left.73 \mathrm{mg} \mathrm{kg}^{-1}\right){ }^{9}{ }^{9} 18 \mathrm{~A}$ batata-doce é uma das tuberosas mais populares do Brasil, sendo consumida na forma assada ou cozida e industrializada na forma de doces. ${ }^{28}$ As concentrações de calistegina $\mathrm{B}_{2}$ neste tubérculo também estão dentro dos níveis reportados na literatura $\left(1,12-19 \mathrm{mg} \mathrm{kg}^{-1}\right){ }^{9,25}$

Calisteginas $A_{3}, B_{1}$ e $B_{2}$ foram encontradas em ambas as cultivares de jilós analisadas, das quais foi possível determinar na cultivar comprido verde claro as concentrações de $\mathrm{A}_{3}\left(6,03 \mathrm{mg} \mathrm{kg}^{-1}\right)$ e $\mathrm{B}_{2}(19,03$ e 23,79 $\left.\mathrm{mg} \mathrm{kg}^{-1}\right)$. Na cultivar jiló morro redondo apenas $\mathrm{B}_{2}$ pôde ser quantificada $\left(15,32\right.$ e $\left.21,81 \mathrm{mg} \mathrm{kg}^{-1}\right)$, o sinal da calistegina $\mathrm{A}_{3}$ ficou abaixo do limite de quantificação.

Novamente é importante ressaltar que essa é a primeira vez em que calisteginas são reportadas em jilós (Solanum aethiopicum L.). Além de serem considerados reservatórios de nutrientes, ${ }^{29}$ os jilós também são popularmente utilizado para fins medicinais com relatos de que apresenta atividade hipoglicemiante. ${ }^{30}$

Embora na literatura não sejam encontrados casos de intoxicação humana por calisteginas via ingestão alimentar, ${ }^{31}$ o conhecimento de teores desses alcalóides em alimentos é importante, principalmente devido aos seus efeitos inibitórios sobre o metabolismo de carboidratos em mamiferos. ${ }^{2}$ 

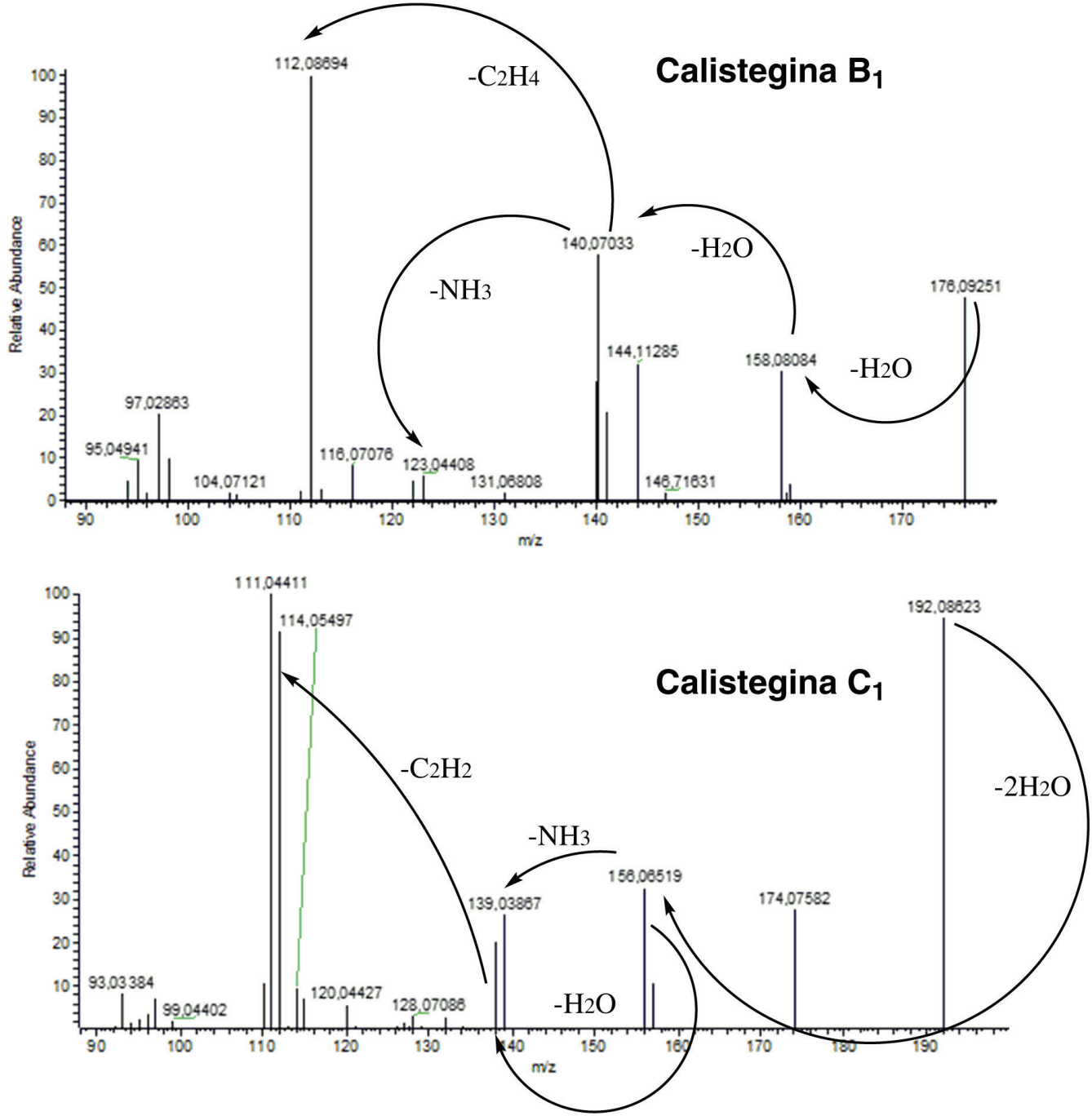

Figura 6. Fragmentação de calisteginas $B_{1}$ e $C_{1}^{21}$ obtidos em CLAE/EM (alta resolução)

Tabela 1. Ocorrência de calisteginas por cultivar detectadas por CG/EM com os tempos de retenção relativos

\begin{tabular}{lccccc}
\hline Parâmetros/Amostras & $\mathrm{A}_{3}$ & $\mathrm{~B}_{1}$ & $\mathrm{~B}_{2}$ & $\mathrm{~B}_{4}$ & $\mathrm{C}_{1}$ \\
\hline Tempo de retenção relativo & 0,75 & 0,84 & 0,88 & 0,81 & 0,94 \\
Batata cv asterix & $\mathrm{x}$ & & $\mathrm{x}$ & $\mathrm{x}$ & \\
Jiló cv morro redondo & $\mathrm{x}$ & $\mathrm{x}$ & $\mathrm{x}$ & & \\
Jiló cv comprido verde claro & $\mathrm{x}$ & $\mathrm{x}$ & $\mathrm{x}$ & & \\
Batata-doce cv BRS rubissol & & $\mathrm{x}$ & $\mathrm{x}$ & & $\mathrm{x}$ \\
Berinjela cv picola & $\mathrm{x}$ & $\mathrm{x}$ & $\mathrm{x}$ & & \\
Tomate cv BRS imigrante & $\mathrm{x}$ & & $\mathrm{x}$ & & \\
\hline
\end{tabular}

Tabela 2. Dados de regressão da curva de calibração em CG/FID

\begin{tabular}{lccc}
\hline Parâmetros & $\mathrm{A}_{3}$ & $\mathrm{~B}_{2}$ & $\mathrm{~B}_{4}$ \\
\hline Coeficiente de determinação $\left(\mathrm{r}^{2}\right)$ & 0,987 & 0,995 & 0,991 \\
Limite de detecção - LD $\left(\mathrm{mg} \mathrm{L}^{-1}\right)$ & 0,03 & 0,01 & 0,02 \\
Limite de quantificação - LQ $\left(\mathrm{mg} \mathrm{L}^{-1}\right)$ & 0,06 & 0,04 & 0,07
\end{tabular}

Tabela 3. Concentração em massa fresca $\left(\mathrm{mg} \mathrm{kg}^{-1}\right)$ das calisteginas $\mathrm{A}_{3}, \mathrm{~B}_{2} \mathrm{e}$ $\mathrm{B}_{4}$ lote $1,(\mathrm{ND}=$ não detectado $)$

\begin{tabular}{lccc}
\hline Cultivares & $\mathrm{A}_{3}$ & $\mathrm{~B}_{2}$ & $\mathrm{~B}_{4}$ \\
\hline Batatas cv asterix & $11,30 \pm 0,50$ & $15,89 \pm 2,42$ & $3,61 \pm 0,50$ \\
Jilós morro redondo & $<\mathrm{LQ}$ & $15,34 \pm 1,47$ & $\mathrm{ND}$ \\
Jilós cv comprido verde claro & $6,03 \pm 1,34$ & $23,79 \pm 1,87$ & $\mathrm{ND}$ \\
Batatas doces cv BRS rubissol & $\mathrm{ND}$ & $10,82 \pm 0,53$ & $\mathrm{ND}$ \\
Berinjelas cv picola & $<\mathrm{LQ}$ & $14,53 \pm 1,54$ & $\mathrm{ND}$ \\
Tomates cv BRS imigrante & $3,53 \pm 0,49$ & $7,59 \pm 0,31$ & $\mathrm{ND}$ \\
\hline
\end{tabular}

Tabela 4. Concentração em massa fresca $\left(\mathrm{mg} \mathrm{kg}^{-1}\right)$ das calisteginas $\mathrm{A}_{3}, \mathrm{~B}_{2} \mathrm{e}$ $\mathrm{B}_{4}$ lote $2,(\mathrm{ND}=$ não detectado $)$

\begin{tabular}{lccc}
\hline Cultivares & $\mathrm{A}_{3}$ & $\mathrm{~B}_{2}$ & $\mathrm{~B}_{4}$ \\
\hline Batatas cv asterix & $15,88 \pm 2,31$ & $19,9 \pm 1,8$ & $9,383 \pm 1,334$ \\
Jilós cv morro redondo & $<\mathrm{LQ}$ & $21,81 \pm 1,88$ & $\mathrm{ND}$ \\
Jilós cv comprido verde claro & $<\mathrm{LQ}$ & $19,03 \pm 2,37$ & $\mathrm{ND}$ \\
Batatas doces cv BRS rubissol & $\mathrm{ND}$ & $11,97 \pm 0,44$ & $\mathrm{ND}$ \\
Berinjelas cv picola & $<\mathrm{LQ}$ & $11,84 \pm 2,58$ & $\mathrm{ND}$ \\
Tomates cv BRS imigrante & $1,94 \pm 1,03$ & $8,05 \pm 0,09$ & $\mathrm{ND}$ \\
\hline
\end{tabular}


Além do fator toxicológico relacionado à ingestão de calisteginas, por serem inibidores de glicosidases, ${ }^{14}$ destaca-se seu potencial como agente hipoglicemiante. Vegetais que contenham esses alcaloides devem ser investigados como possíveis componentes de uma dieta que evite aumento acentuado de glicose no sangue após uma refeição rica em carboidratos. ${ }^{2}$ Em camundongos diabéticos, esses compostos reduziram para concentrações normais os níveis de glicose no sangue e parâmetros lipídicos, evidenciando que esses compostos sejam potentes agentes antidiabéticos com efeitos anti-hiperglicêmicos. ${ }^{32}$

\section{CONCLUSÕES}

Calisteginas foram determinadas em cultivares de hortaliças comuns na mesa dos brasileiros, cinco foram detectadas em todo o conjunto amostral $\left(\mathrm{A}_{3}, \mathrm{~B}_{1}, \mathrm{~B}_{2}, \mathrm{~B}_{4}\right.$ e $\left.\mathrm{C}_{1}\right)$, três tiveram suas concentrações determinadas $\left(\mathrm{A}_{3}, \mathrm{~B}_{2}\right.$, e $\left.\mathrm{B}_{4}\right)$, apresentando valores similares àqueles descritos na literatura. Esses alcaloides foram encontrados em todas as espécies analisadas, com destaque para a calistegina $B_{2}$ que foi quantificada em todas as amostas. Este é o primeiro relato da ocorrência de calisteginas $\left(A_{3}, B_{1}\right.$ e $\left.B_{2}\right)$ em jilós (Solanum aethiopicum $\mathrm{L}$.), detectadas nas duas cultivares analisadas.

\section{MATERIAL SUPLEMENTAR}

Em http://quimicanova.sbq.org.br, podem ser encontradas, as estruturas das calisteginas derivatizadas, cromatogramas CG/EM das amostras, espectros de massas dos padrões e as curvas analíticas, disponíveis com acesso livre.

\section{AGRADECIMENTOS}

Ao IFRO, UFRJ/IPPN, CNPq, CAPES e FAPERJ pelo apoio institucional e financeiro. Ao Professor Rafael Garrett da Costa coordenador do Laboratório de Metabolômica (LabMeta-LADETEC/ IQ-UFRJ) pelas análises por CLAE/EM.

\section{REFERÊNCIAS}

1. Biastoff, S.; Dräger, B. Em The Alkaloids: Chemistry and Biology. Cordell, ed.; Academic Press: San Diego, 2007, cap. 2.

2. Jocković, N.; Fischer, W.; Brandsch, M.; Brandt, W.; Dräger, B.; J. Agric. Food Chem. 2013, 61, 5550.

3. Goldmann, A.; Milat, M. L.; Ducrot, P. H.; Lallemand, J. Y.; Maille, M.; Lepingle, A.; Charpin, I.; Tepfer, D.; Phytochemistry 1990, 29, 2125.

4. Asano, N.; Kato, A.; Oseki, K.; Kizu, H.; Matsui, K.; Eur. J. Biochem. 1995, 229, 369.

5. Asano, N.; Oseki, K.; Tomioka, E.; Kizu, H.; Matsui, K.; Carbohydr. Res. 1994, 259, 243.

6. Brock, A.; Bieri, S.; Christen P.; Dräger B.; Phytochemistry 2005, 66, 1231.
7. Brock, A.; Herzfeld, T.; Paschke, T.; Koch, M.; Draeger, B.; Phytochemistry 2006, 67, 2050.

8. Asano, N.; Strusovskaya, O. G.; Kosyakov, D. S.; Pokryshkin, S. A.; Gavrilin, M. V.; Mudretsova, J. V.; Chemistry of plant materials 2014, 2, 207.

9. Asano, N.; Kato, A.; Matsui, K.; Watson, A. A.; Nash, R. J.; Molyneux, R. J.; Winchester, B.; Glycobiology 1997, 7, 1085.

10. Arcella, D.; Altieri, A. EFSA J. 2018, 16, 5160.

11. Mulder, P. P.; de Nijs, M.; Castellari, M.; Hortos, M.; MacDonald, S.; Crews, C.; Stranska, M.; EFSA Supporting Publications 2016, 13, 1140.

12. Richter, U.; Sonnewald, U.; Dräger, B.; J. Exp. Bot. 2007, 58, 1603.

13. Friedman, M.; Roitman, J. N.; Kozukue, N.J.; Agric. Food Chem. 2003, 51, 2964.

14. Bourebaba, L.; Sullini, G.; Mendiola, J. A.; Bourebaba, Y.; Deghima, A.; Oukil, N.; Bedjou, F.; Ind. Crops Prod. 2016, 89, 316.

15. Griffiths, D.; W., Shepherd, T.; Stewart, D.; Agric. Food Chem. 2008, $56,5197$.

16. Jocković, N.; Tese de Doutorado, Martin-Luther-Universität HalleWittenberg, Alemanha, 2003.

17. Petersson, E. V.; Arif, U.; Schulzova, V.; Krtková, V.; Hajšlová, J.; Meijer, J.; Sitbon, F.; J. Agric. Food Chem. 2013, 61, 5893.

18. Dräger, B.; Phytochem. Anal. 1995, 61, 37.

19. Pizzutti, I. R.; de Kok, A.; Cardoso, C. D.; Reichert, B.; de Kroon, M.; Wind, W.; da Silva, R. C.; J. Chromatogr. A 2012, 16, 1251.

20. Xia, Y. G.; Sun, H. M.; Wang, T. L.; Liang, J.; Yang, B. Y.; Kuang, H. X.; Molecules 2018, 23, 1284.

21. Romera-Torres, A.; Romero-González, R.; Vidal, J. L. M.; Frenich, A. G.; J. Chromatogr. A 2018, 1576, 51.

22. Nash, R. J.; Rothschild, M.; Porter, E. A.; Watson, A. A.; Waigh, R. D.; Waterman, P. G.; Phytochemistry 1993, 34, 1281.

23. Ribani, M.; Bottoli, C. B. G.; Collins, C. H.; Jardim, I. C. S. F.; Melo, L. F. C.; Quim. Nova. 2004, 27, 771.

24. Bekkouche, K.; Daali, Y.; Cherkaoui, S.; Veuthey, J. L.; Christen, P.; Phytochemistry 2001, 58, 455.

25. Schimming, T.; Tofern, B.; Mann, P.; Richter, A.; Jenett-Siems, K.; Dräger, B.; Eich, E.; Phytochemistry 1998, 49, 1989.

26. Keiner, R.; Dräger, B.; Plant Science 2000, 150, 171.

27. Das, M.; Barua, N.; Int. J. Green Pharm. 2013, 7, 274. Phytochemistry 1993, 34, 1281

28. Leonel, M.; Cereda, M. P.; Food Sci. Technol. 2002, 22, 65.

29. Alexandre, E. R.; Herculano, L. M.; da Silva, J. M.; de Oliveira, S. M. A.; Pesqui. Agropecu. Bras. 2014, 49, 930.

30. Santos, M. M.; Nunes, M. G. S.; Martins, R. D.; Rev. Bras. Plant. Med. 2010, 14, 327.

31. Binaglia, M.; Baert, K.; Schutte, M.; Serafimova, R.; EFSA J. 2019, 17, 5574.

32. Bourebaba, L.; Saci, S.; Touguit, D.; Gali, L.; Terkmane, S.; Oukil, N.; Bedjou, F.; Biomed. Pharmacother. 2016, 82, 337. 